\title{
Apprendenti universitari e profili di competenza nella scrittura accademica
}

\author{
Paola Polselli \\ Università di Bologna \\ paola.polselli@unibo.it
}

\author{
Alice Fatone \\ Universitat de València \\ alice.fatone@uv.es
}

\section{Riassunto}

Nell'ambito delle ricerche sulle competenze linguistiche degli studenti universitari in Italia, il contributo indaga le pratiche di comunicazione scritta e le relative difficoltà espresse da studenti di un corso di recupero OFA in corsi di laurea triennale. L'indagine è parte di un progetto di rilevazione più ampio ed è stata realizzata somministrando un questionario informatizzato. I dati raccolti permettono di sviluppare alcune riflessioni utili sul profilo linguistico-comunicativo di apprendenti dalle competenze definite "fragili"; la loro autorappresentazione in termini di biografia linguistica; i bisogni linguistico-comunicativi espressi in rapporto ai compiti di scrittura, e il senso di autoefficacia percepita in rapporto alle abilità di scrittura funzionale richieste. Nell'insieme, il divario tra il panorama linguistico delle matricole in esame e quello del nuovo contesto di studio avallano le indicazioni circa la necessità di prevedere una specifica formazione linguistica in ambito universitario superando l'impostazione rimediale per una prospettiva più strutturale e continuativa.

Parole chiave: educazione linguistica; scrittura all'università; studenti e senso di autoefficacia; biografia linguistica; profilo accademico degli apprendenti.

\section{Abstract. College learners and academic writing proficiency profiles}

In the context of research on the linguistic competences of university students in Italy, this contribution investigates the written communication practices and related difficulties expressed by students in an OFA remedial course of BA degree programmes. The survey was carried out by administering a computerised questionnaire and is part of a wider university project. It aims to define the linguistic-communicative profile of these learners, their representation of their linguistic abilities required in the academic environment. On the whole, the collected data highlight the gap between the linguistic landscape of newly enrolled students and that of the new study context endorsing the indications about the need to provide specific language training in the university environment and to overcome the remedial approach for a more structural educational perspective.

Keywords: language education; writing at university; students and sense of self-efficacy; language biography; learners' academic profile. 


\section{Competenze linguistiche e scrittura accademica ${ }^{1}$}

All'inizio degli anni Novanta, introducendo uno dei primi studi sistematici sulle questioni poste dall'educazione linguistica degli studenti universitari (Lavinio \& Sobrero, 1991), Sobrero avanzava l'ipotesi che le carenze di scrittura di laureandi e laureati fossero "da addebitare soprattutto alla mancanza di pratiche di scrittura 'assistita' che caratterizzino tutto l'arco degli studi universitari" (Sobrero, 1991, p. 5) e auspicava un'offerta formativa caratterizzata da maggiori occasioni di scrittura legate a processi di ricerca e a contenuti disciplinari in un'ottica trasversale alle varie discipline. Per definire un'efficace formazione universitaria sulla scrittura, concludeva, l'università avrebbe dovuto assumersi le "pesanti responsabilità che le competono" (Sobrero, 1991, p. 6).

Da allora, gli studi sul discorso accademico hanno visto svariati contributi che si sono soffermati sull'analisi della dimensione comunicativa orale e, soprattutto, sulla comprensione e produzione scritta di studenti universitari italiani considerandone campioni di lingua tratti da testi funzionali allo studio accademico o da comunicazioni formali (tra gli altri, Andorno, 2014; Brusco et al., 2014; Ciliberti \& Anderson, 1999; Desideri \& Tessuto, 2011; Fiorentino, 2015; Fusari \& Luporini, 2016; Grandi, 2018; Lavinio, 2011; Sposetti, 2008; Valentini, 2002; Voghera, Giordano \& Guerriero, 2009). Altre ricerche hanno riguardato in modo specifico la consapevolezza metalinguistica degli studenti di area umanistica, futuri insegnanti di materie linguistiche (Lavinio, 2011; Viale, 2011); di recente, il campo d'indagine si è esteso oltre le produzioni in L1 fino a comprendere quelle in L2 e l'analisi di testi scritti da studenti stranieri, attori della nuova università internazionalizzata (Lubello, 2019; Lubello \& Nobili, 2019; Martari, 2019; Pugliese \& Della Putta, 2017, 2020). In proposito un dato che emerge nella realtà accademica coeva riguarda proprio l'eterogeneità dei profili di apprendente: anche solo considerando i cosiddetti "studenti stranieri", Grassi (2018) ne distingue almeno tre (italofoni nativi con scolarizzazione in italiano, italofoni non nativi con scolarizzazione e diploma all'estero, italofoni non nativi con diploma italiano).

Se guardiamo agli studi sviluppati nell'arco temporale di questo trentennio, osserviamo, dunque, due tratti distintivi del dibattito: da una parte, si rileva il perdurare delle denunce/aspettative circa una (in)compiuta padronanza delle competenze necessarie per il discorso accademico al termine della scuola secondaria; dall'altra, emerge il continuo lavoro su dati empirici per documentare le debolezze linguistiche dei giovani italiani e chiedere di affrontare in modo sistematico la formazione linguistica nel terzo ciclo.

Nel primo caso, oggetto del confronto è la responsabilità di sviluppare le "competenze chiave" (raccomandazione europea 2006/962/CE) e in particolare la "competenza alfabetica funzionale" nella lingua di scolarizzazione (raccomandazione 2018/C 189/01). Infatti, se molti accademici la attribuiscono

1. Il contributo e la relativa ricerca sono stati realizzati in collaborazione tra le autrici. Nella stesura, Paola Polselli ha scritto i parr.1, 2, 5, 6; Alice Fatone ha scritto i parr. 3, 4. I parr. 7,8 sono a cura di entrambe. 
alla scuola dell'obbligo, molti linguisti la includono nei doveri di formazione linguistica che dovrebbero caratterizzare tutti i livelli d'istruzione in un'ottica di apprendimento permanente. Di tale contrapposizione è stata un esempio emblematico la diatriba conseguente alla lettera aperta firmata da 600 docenti universitari che nel 2017 ha denunciato le incompetenze degli studenti universitari imputandole al mancato raggiungimento degli obiettivi formativi della scuola dell'obbligo. L'evento, che ha guadagnato gli onori della cronaca grazie a una vasta eco mediatica, ha visto la risposta di molti accademici di area linguistica, in primis di Lo Duca (2017), che hanno affermato come una prospettiva realistica sui fatti di lingua debba rifuggire da nostalgie anacronistiche e richieda proposte specifiche e una lettura più attenta delle "spinte che le forze in gioco nella realtà sociale esercitano sulla lingua e che finiscono per modificare il modo in cui parliamo e scriviamo" (De Santis \& Fiorentino, 2018, p. 9).

In quest'ottica, l'offerta formativa dovrebbe essere improntata a un approccio olistico ed ecologico che intervenga in riferimento al più ampio quadro delle esperienze linguistiche e testuali degli studenti perché la "separatezza tra lavoro grammaticale e lavoro sui testi non permette di creare una grammatica capace di seguire i movimenti della lingua nei vari generi e contesti” mentre appare fondamentale operare affinché gli studenti possano costruirsi "una grammatica attiva [...] modellata sui bisogni comunicativi reali e quindi in continua crescita e movimento", superare gli "steccati disciplinari (e accademici)" (Voghera et al., 2009, p. 107). È dunque opportuno considerare le ricerche svolte come base di attente analisi dei bisogni e stimolo per insegnamenti che superino i tradizionali metodi didattici trasmissivi (cfr. Lavinio, 2011; Pagliara, 2018).

Circa i dati empirici della ricerca sulla scrittura all'università, come abbiamo visto nella rassegna delle principali ricerche svolte, oggi disponiamo di una buona base conoscitiva i cui dati provengono sostanzialmente dal "filone che descrive lo stato delle (in)competenze linguistiche degli studenti" (Pugliese, 2021, p. 178), una base di dati costituita da corpora di elaborati scritti raccolti all'inizio, nel corso o al termine di un percorso di studi universitario. Di contro, sono scarsi i dati relativi a come gli studenti universitari vedono la scrittura e vivono le pratiche comunicative scritte. In proposito, è rilevante il concetto di rapport à l'écriture (Barré-De Miniac, 2002), definito in quell'area della ricerca francofona che indaga la rete dei legami esistenti tra lo studente-scrivente e l'attività di scrittura, vista come pratica individuale e sociale, esperienziale e accademica, situata localmente e determinata culturalmente. Barré-De Miniac sottolinea, oltre alla rilevanza dei fattori cognitivi, l'incidenza degli aspetti affettivi, culturali e sociali e concettualizza il rapporto che un apprendente ha con la scrittura in 4 dimensioni: investimento nella scrittura; opinioni e atteggiamenti rispetto alla scrittura; concezione della scrittura e del suo apprendimento; modalità di investimento o "la manière dont les scripteurs parlent de leur écriture, et plus exactement de leurs procédures et de leurs démarches, tant en matière d'écriture proprement dite qu'en ce 
qui concerne leur apprentissage de l'écrit." (Barré-De Miniac, 2002, p. 37). Indagare in tale direzione permette dunque sia di rilevare alcuni tratti trasversali del contesto educativo e storico-culturale sia di operare nel senso di un approfondimento individualizzato dello sviluppo delle competenze di scrittura nei singoli apprendenti nell'ottica dello sviluppo di littéracies di livello universitario. In altri termini, rilevare le prospettive degli studenti nella fase iniziale di un corso di scrittura non permette soltanto di raccogliere informazioni utili, fornisce anche alcune indicazioni fondamentali "per l'accompagnamento alla scrittura" come strumento di costruzione del sapere disciplinare e rappresenta "una buona illustrazione della dinamica interattiva tra ricerca e pratica didattica” sulla scrittura accademica (Desoutter, 2021, p. 24).

\section{L'indagine}

Come ricorda Guerriero (2021), saper scrivere è un'abilità multidimensionale in cui intervengono sia la capacità di costruire un testo definendolo nei suoi diversi livelli microlinguistici, macrolinguistici e pragmatici (cioè nei suoi aspetti semantici, sintattici e semantico-testuali; relativi a finalità transazionali e relazionali, ai riceventi e al quadro sociale della comunicazione scritta), sia la capacità di gestire a livello procedurale le diverse fasi che caratterizzano il processo di scrittura (come ideare, pianificare, redigere e revisionare un testo). Che il compito della scrittura non sia banale, lo ricorda Piemontese (2002 (2021), p. 33) allorché sottolinea "la necessità di sviluppare negli studenti una maggiore consapevolezza nel perseguire [...] gli obiettivi che essi vogliono dare al testo" ed evidenzia l'importanza della rappresentazione che gli apprendenti hanno di tale attività. "Obiettivo della didattica della scrittura è dare agli studenti la percezione della scrittura come problem solving per cui sono necessarie autodisciplina, capacità di valutazione, diagnosi e riflessione". Consapevolezza, percezione, capacità riflessiva e di auto/valutazione sono appunto alcune delle parole centrali nell'indagine che presentiamo.

Progettare un'offerta di formazione linguistica efficace che coadiuvi lo sviluppo di competenze comunicativo-accademiche nell'apprendimento dei saperi disciplinari in una data popolazione studentesca presuppone infatti di operare a partire da un'analisi che renda conto dello scarto osservabile tra gli usi comunicativi diffusi e le capacità di scrittura richieste in contesto universitario. In questa prospettiva, la nostra ricerca mirava a delineare il profilo linguistico, il rapporto con la scrittura e le relative pratiche da parte degli studenti universitari nonché la loro percezione dei propri bisogni formativi.

I dati che discuteremo fanno parte di un progetto di indagine più esteso e tuttora in corso: è stato intrapreso nell'a.a. 2017/2018 e comprende i questionari di circa cento studenti universitari di vario tipo. Si tratta di studenti italiani con e senza OFA, studenti universitari internazionali che studiano in Italia o che partecipano a programmi di scambio internazionale, apprendenti della lingua italiana in un'università spagnola. In particolare, il corpus di dati qui analizzato si sofferma su apprendenti che non hanno raggiunto la 
soglia minima prevista nel test d'accesso all'università, cioè studenti che intraprendono un corso di studi all'università con un "debito formativo" (OFA Obbligo Formativo Aggiuntivo) e le cui competenze linguistiche di base sono state giudicate insufficienti per l'accesso a un corso di studi di laurea triennale.

A livello nazionale, l'istituzione degli Obblighi Formativi Aggiuntivi $(\mathrm{OFA})^{2}$ ha risposto all'esigenza di allineare le percentuali dei laureati italiani a quelli europei, di ridurre i tassi di abbandono nei primi anni di studio universitario e di colmare i dislivelli lamentati dai docenti rispetto alle competenze linguistico-comunicative richieste dal discorso accademico e disciplinare. Concettualmente, però, un'offerta formativa nell'area linguistica che ponga al centro il concetto di "fragilità" piuttosto che quello dell'eterogeneità delle competenze oggi riscontrabile in ingresso nel mondo universitario e delle connesse implicazioni formative per il terzo ciclo educativo ha evidenti ricadute didattiche. La dimensione "rimediale", infatti, tradisce il respiro breve dell'offerta di formazione linguistica a livello universitario e incide negativamente sulla motivazione all'apprendimento specifico anche in quei corsi di studi in cui tali competenze dovrebbero apparire professionalmente cruciali (cfr. BarréDe-Miniac, 2003, Pugliese, 2021).

Il corpus consta delle risposte a un questionario informatizzato di 44 studentesse con OFA di due corsi di laurea triennale nell'a.a. 2018/2019. ${ }^{3}$ Le domande da cui è partita l'indagine sono le seguenti:

- qual è il panorama linguistico di questi apprendenti? E quali sono gli usi delle (varietà di) lingue conosciute?

- quali attività di scrittura sono frequenti nella pratica quotidiana? Qual è il rapporto con la scrittura e qual è il senso della propria autoefficacia? - quali testi sono percepiti come difficili e perché? Quali argomenti di lavoro propongono?

Il questionario informatizzato proposto è stato organizzato in 4 sezioni: dopo una prima parte anagrafica, la seconda sezione mira a delineare la biografia linguistica dell'apprendente e a ricostruirne il panorama degli usi linguistici ricorrenti o saltuari; la terza indaga le pratiche scritte e l'ultima richiede di valutare diversi compiti di scrittura esplicitando la propria percezione di autoefficacia (Bandura, 1994) e le coordinate del proprio rapporto con la scrittura.

Una precisazione utile per inquadrare le risposte fornite riguarda il momento in cui si è svolta l'indagine. Il questionario è stato somministrato nel febbraio $2019,{ }^{4}$ cioè in un'epoca in cui le matricole avevano realizzato le prime esperienze di studio in ambito universitario e avevano già avuto l'opportunità di impostare delle abitudini di studio e di scrittura relative ai compiti

2. V. in particolare il D.M. n. 509 del 3/11/1999 e il D.M. n. 270 del 22/10/2004.

3. Corsi di Laurea Triennale in Educatore sociale e culturale e in Educatore nei servizi per l'infanzia del Dipartimento di Scienze dell'Educazione dell'Università di Bologna (sede di Bologna).

4. Per comprendervi gli studenti trasferitisi da altri corsi di studio, i corsi OFA si sono svolti nel II semestre. 
comunicativi previsti in contesto accademico. In questo senso, le risposte fornite nel questionario non rendono conto soltanto del profilo delle matricole, ma consentono anche di rilevare alcune routine e usi della scrittura in atto nelle attività di studio a metà del primo anno accademico.

\section{Le autobiografie linguistiche nel questionario}

Le autobiografie linguistiche (AL) rappresentano il riferimento soggiacente alla prima parte del questionario in cui si vuole ricostruire il profilo linguistico delle rispondenti. L'indagine non ambisce all'ampiezza e alla profondità praticata con strumenti come l'AL proposta nel Portfolio Europeo delle Lingue (PEL) ma vuole essere un'occasione di riflessione sulle abitudini e le competenze linguistiche delle rispondenti e sulle loro pratiche comunicative. Analizzare le proprie competenze linguistiche trasversali alle diverse lingue conosciute, infatti, assume ricadute positive sulle capacità metalinguistiche. Per questo, rappresentare la gamma linguistica delle rispondenti ha un valore profondo non solo per l'AL ma anche in relazione con il successivo corso OFA. Prima di discutere $\mathrm{i}$ dati relativi alla prima sezione del questionario, dove sono indagate le AL delle 44 apprendenti, consideriamo l'importanza di questo strumento a cui la prima parte del questionario fa riferimento.

Gli studi di Pineau (1983) hanno evidenziato le potenzialità dell'AL come strumento formativo studiato e sperimentato soprattutto nell'ambito delle scienze dell'educazione e della formazione degli adulti.

Nell'ultimo decennio, sul territorio nazionale e internazionale, l'ampia letteratura (tra gli altri Lambert, 2005; Lüdi, 2005; Molinié, 2006; Perregaux, 2007; Van den Avenne, 2005) e numerosi progetti europei, in particolare in contesti francofoni e tedescofoni, evidenziano come l'uso di questo strumento linguistico sia ormai un campo di indagine ricco e in espansione, tanto per nuove prospettive di ricerca che dal punto di vista applicativo in contesto educativo-formativo con diverse tipologie di pubblici.

In Italia, l'AL, nel contesto dell'apprendimento/insegnamento delle lingue, ha iniziato a diffondersi in particolare grazie al PEL, considerato un efficace strumento (auto) valutativo di analisi della propria esperienza linguistica, capace di riflettere e descrivere le modalità di apprendimento e le esperienze di contatto interculturale in contesto formale e non.

In ambito anglo-americano si intendono le AL come narrazioni autobiografiche che presentano l'esperienza degli apprendenti di una L2 o di parlanti che vivono a contatto con più di una lingua. Esse sono usate a scopi di ricerca come risorsa per la comprensione dei processi di acquisizione e dell'uso di una lingua seconda (Kramsch, 2004). In ambito europeo, invece, prevalgono le definizioni di autobiografia/biografia linguistica, in accordo con il PEL, o di "racconto delle lingue", che si soffermano maggiormente sull'importanza dell'aspetto narrativo e della prospettiva del soggetto narrante (Cognigni, 2014, p. 3). 
Il legame tra madrelingua e identità è intricato e inscindibile (Sofia $\&$ Favero, 2018, p. 50) e, come hanno sottolineato diverse ricerche (tra gli altri Cognigni, 2014; Levy, 2009; Gohard-Radenkovic \& Rachédi, 2010; Sofia \& Favero, 2018), le AL, oltre a valorizzare il patrimonio linguistico personale in un'ottica metalinguistica, sono efficaci per la (ri)costruzione identitaria e per l'emersione della dimensione emotivo-affettiva nel rapporto con le diverse lingue e culture che compongono la competenza plurilingue e pluriculturale di ciascuno.

Nello specifico Cognigni (2014) sintetizza due fondamentali funzioni dell'AL: una diagnostica o conoscitiva e un'altra legata alla possibilità di elicitare le rappresentazioni sociali degli alunni circa le proprie lingue-culture.

Nel contesto di classi plurilingui e pluriculturali, soprattutto, il docente può avvalersi della narrazione scritta o orale per approfondire la conoscenza dei repertori plurilingui degli apprendenti, i contesti d'uso dei diversi codici e i metodi didattici legati alla storia di acquisizione/apprendimento linguistico lungo il percorso di vita.

La condivisione in aula dei racconti delle proprie lingue-culture attiva una maggiore consapevolezza linguistica (Language Awareness) che permette all'apprendente di rendersi conto dell'esistenza di lingue differenti dalla propria e di prendere coscienza di possedere più lingue. Il discente, inoltre, si rende conto del processo di apprendimento linguistico (Language Learning Awareness) e della variazione linguistica (Variation Awareness) intesa come consapevolezza della presenza di diverse varietà di una medesima lingua e dei loro diversi usi sociali.

L'AL diventa, quindi, strumento conoscitivo e operativo (Sofia \& Favero, 2018), capace di creare un ponte tra studente e docente e tra lo studente e il repertorio di lingue possedute o presenti nel gruppo di apprendenti.

$\mathrm{Nel}$ nostro caso, il questionario proposto serviva anche ad orientare il lavoro dell'insegnante, a raccogliere informazioni sui bisogni formativi, a conoscere le apprendenti e avere informazioni utili ai fini del corso.

\section{Il profilo linguistico delle apprendenti}

Analizziamo ora i dati delle prime sezioni (A e B) del questionario. Inizialmente, le rispondenti precisano la propria madrelingua: sono per il $92 \%$ di madrelingua italiana e per il restante $8 \%$ di madrelingua albanese, araba, filippina e francese.

Nella sezione A del questionario "l'italiano e le altre lingue" si chiedeva di indicare il numero di lingue conosciute scegliendo tra 1-2 o 3-4 lingue/dialetti. Nel grafico 1 si mostra la distribuzione percentuale in termini di ampiezza di lingue conosciute oltre quella materna.

I dati riflettono scelte di politica linguistica del sistema scolastico italiano che ha previsto, in seguito alla legge 53/2003, l'insegnamento obbligatorio dell'inglese dal primo anno della scuola primaria e il successivo bilinguismo 


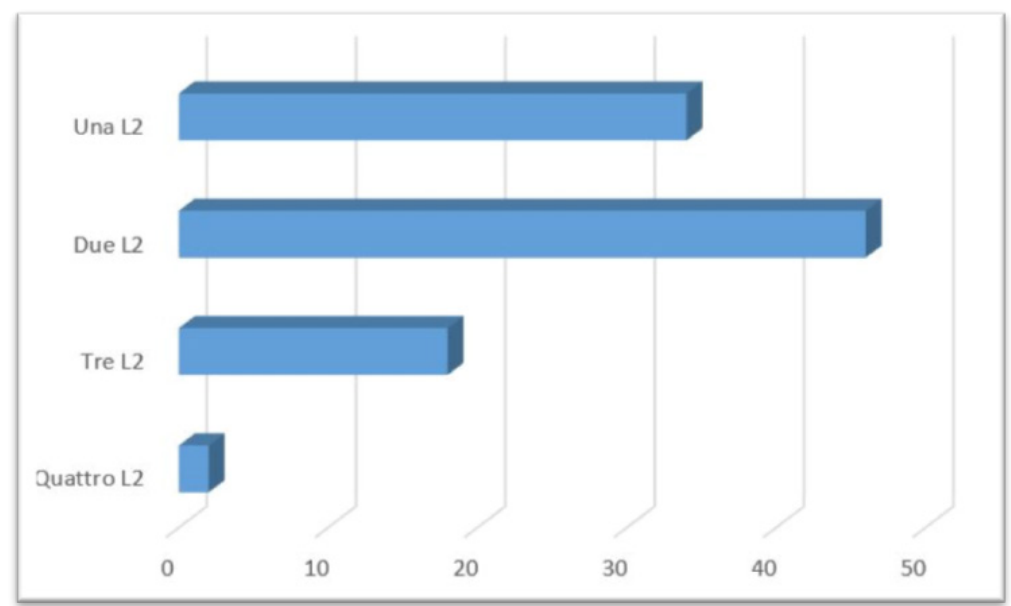

Grafico 1- Distribuzione percentuale del numero di L2 conosciute oltre alla L1

degli Istituti superiori di primo e secondo grado. Chi parla due lingue specifica di non usare più una lingua perché "la usavo a scuola e ora non la uso più" (in 3 occorrenze: questionario 3, 5, 19). Un altro elemento che scaturisce dai dati è una certa confusione nella distinzione tra L1 e L2. In 4 occorrenze, le rispondenti descrivono come L2 la loro madrelingua ed è interessante osservare che, per la metà di questi casi, si tratta di madrelingue diverse dall'italiano. È difficile interpretare questa scelta, forse si potrebbe ipotizzare l'esigenza di rappresentare la competenza nella propria madrelingua.

La sezione B "le lingue del mio panorama" definiva in dettaglio le competenze linguistico-comunicative delle apprendenti. I dati raccolti, presentati nella tabella 1 , intendevano fotografare le lingue conosciute dalle rispondenti oltre alla L1. In termini assoluti la prima L2 nota è prevedibilmente l'inglese, la seconda lo spagnolo, la terza il francese. Con una sola occorrenza, ci sono poi altre L2 come l'albanese, l'italiano e un dialetto.

\begin{tabular}{|l|l|l|l|l|}
\hline Lingue seconde (L2) & Prima L2 & Seconda L2 & Terza L2 & Quarta L2 \\
\hline Albanese & 1 & & & \\
\hline Arabo & & 1 & 1 & \\
\hline Dialetto & 1 & 2 & & 1 \\
\hline Francese & 6 & 7 & 1 & \\
\hline Inglese & 29 & 4 & 3 & \\
\hline Italiano & 1 & 2 & & \\
\hline Spagnolo & 6 & 5 & 3 & \\
\hline Tedesco & & & & \\
\hline
\end{tabular}

Tabella 1- Numero di occorrenze delle L2 conosciute 
Circa la seconda L2 conosciuta, il ventaglio è molto più ampio e comprende diverse lingue: il francese, l'inglese, lo spagnolo, l'italiano, il dialetto e l'arabo. Continuando un'analisi in termini assoluti, osserviamo che il 18\% dichiara di conoscere una terza lingua e che una sola studentessa dichiara di conoscere una quarta L2 (un dialetto).

Ricostruire il panorama delle lingue ha in Italia un particolare interesse alla luce del plurilinguismo storico che affianca all'italiano la presenza di un dialetto imparato e parlato in contesto famigliare. Per descrivere questa situazione nella nostra indagine, osserviamo i dati disaggregati: come presupposto, il dialetto è nel $14 \%$ dei casi parte costitutiva della biografia linguistica delle rispondenti e, in un caso, risulta essere l'unica lingua parlata oltre la madrelingua. Nel 7\% dei casi le intervistate specificano che si tratta del dialetto romagnolo. I livelli di competenza raggiunti sono diversi: elementare in 3 casi, intermedio in uno e avanzato in 2 . Il dialetto è appreso da piccole e continua ad essere usato in famiglia per comunicare.

La seconda domanda indagava i momenti d'uso della prima L2 conosciuta. Per lo più, le rispondenti indicano usi diversificati che riassumiamo nel grafico 2 dove predominano come ambiti d'uso il viaggio, il tempo libero e la famiglia.

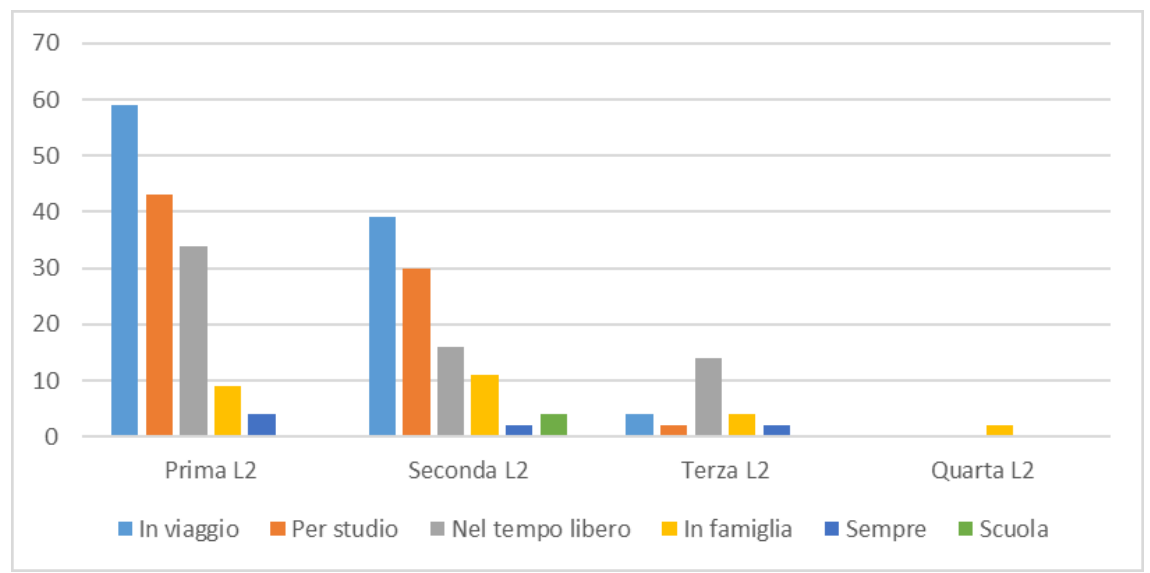

Grafico 2 - Distribuzione percentuale degli ambiti d'uso delle L2 conosciute

Nello specifico, le risposte libere indicano in 3 occorrenze che la prima L2 era usata alle scuole superiori, in un'occorrenza era usata "per test" (q. 10), "al termine delle medie e poi non ho più avuto occasione" (q. 14), "l'ho studiata quando avevo 8 anni" (q. 41) "la uso quando devo" (q. 42). L'inglese, prima lingua conosciuta, è usato come lingua franca in viaggio con una competenza comunicativa informale e senza un apprendimento più strutturato o in contesti di studio (dove spesso l'apprendimento della lingua inizia, continua e poi si ferma). L'apprendimento informale è quello della lingua usata nel tempo libero dove dominano lingue come l'inglese e lo spagnolo per usi legati al web (in particolare serie televisive su piattaforme digitali) e/o alla musica. Le occasioni 
d'uso affettivo e intimo della lingua si limitano alla descrizione della lingua madre o del dialetto.

Circa gli ambiti d'uso, una studentessa dichiara di "usare poco" (q.17) la seconda L2. La terza L2 (che, ricordiamo, è posseduta solo dal 18\% delle rispondenti) è usata prevalentemente nel tempo libero. La rispondente che possiede una quarta L2 dichiara di usarla solo in famiglia.

$\grave{E}$ interessante evidenziare come le seconde e terze L2 vengano usate preferibilmente per il tempo libero o in viaggio e inoltre come il contesto scolastico sia sempre più relegato a un passato in cui la lingua lì appresa non trova altri contesti d'uso. Un apprendimento troppo focalizzato sulla competenza linguistica e poco incentrato sulle occasioni d'uso comunicativo mostra fragilità perché le lingue non evolvono. Questi dati sottolineano che il focus esclusivo sulla competenza linguistica nella formazione, a scapito della più ampia competenza comunicativa e della competenza pragmatica ${ }^{5}$, può più facilmente portare alla perdita delle conoscenze linguistiche conosciute.

La terza domanda indagava gli usi linguistici di ogni lingua conosciuta. Il grafico 3 riassume i risultanti delle dichiaranti.

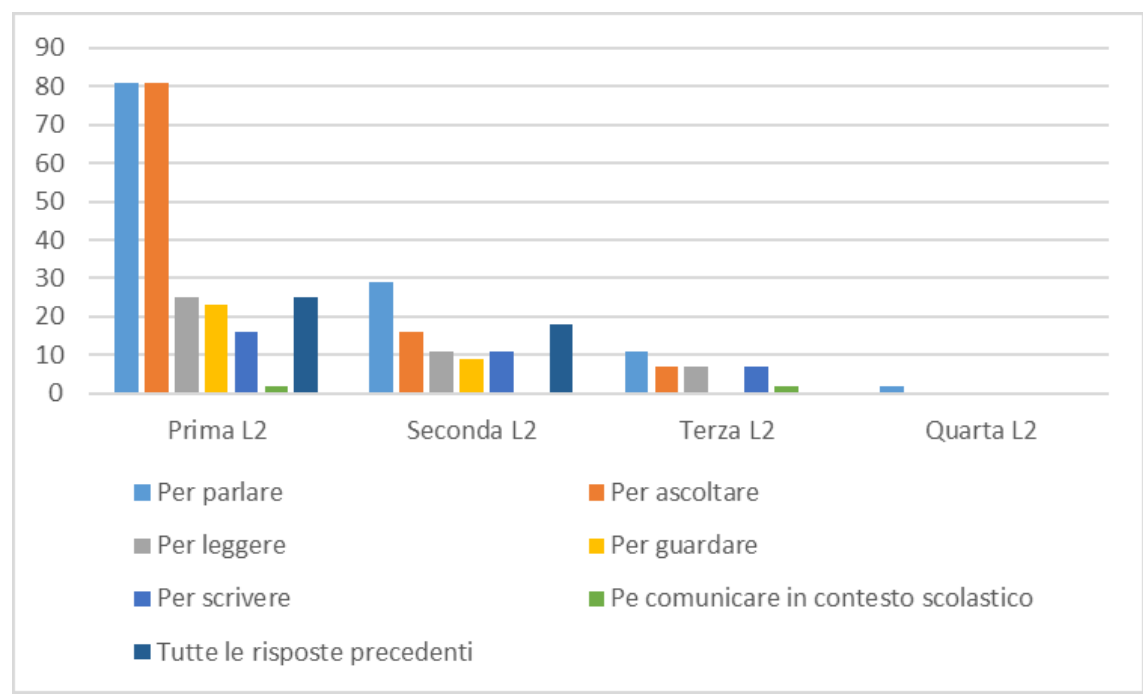

Grafico 3- Distribuzione percentuale degli usi linguistici prevalenti delle L2

I dati presentati nel grafico 3 continuano ad essere allineati con le risposte precedenti: le lingue, anche le prime conosciute, hanno obiettivi comunicativi

5. Da questo momento ci si riferisce alla competenza comunicativa e a quella pragmatica nell'ottica del Quadro Comune Europeo delle Lingue (cfr. pp. 140-141 QCER, 2002) che presenta scale di descrittori per gli aspetti della competenza linguistico-comunicativa organizzate in competenza linguistica, competenza sociolinguistica e competenza pragmatica (flessibilità, turni di parola, sviluppo tematico, coerenza e coesione, precisione e fluenza). 
molto più legati all'oralità e alla velocità (parlare, ascoltare, guardare) che a una riflessione più lenta e riflessiva (leggere, scrivere).

La quarta domanda interrogava sui luoghi d'uso e gli interlocutori tipici e proponeva tra le possibili scelte: persone straniere, il web, i parenti, l'università, il contesto scolastico e il lavoro (grafico 4).

Le lingue per le rispondenti sono legate ad apprendimenti informali, incidentali e l'uso nel contesto accademico e scolastico o formale è limitato a un quarto delle rispondenti e alla prima L2 (che è per lo più l'inglese). Le lingue, ancora una volta, sono abbandonate nel corso del tempo (sono materia di studio fino alla scuola secondaria) e poi sono ad esse affidati ruoli comunicativi informali (in famiglia, a casa o con amici) o fini ludici, di divertimento, di informazione privata (usate nel web).

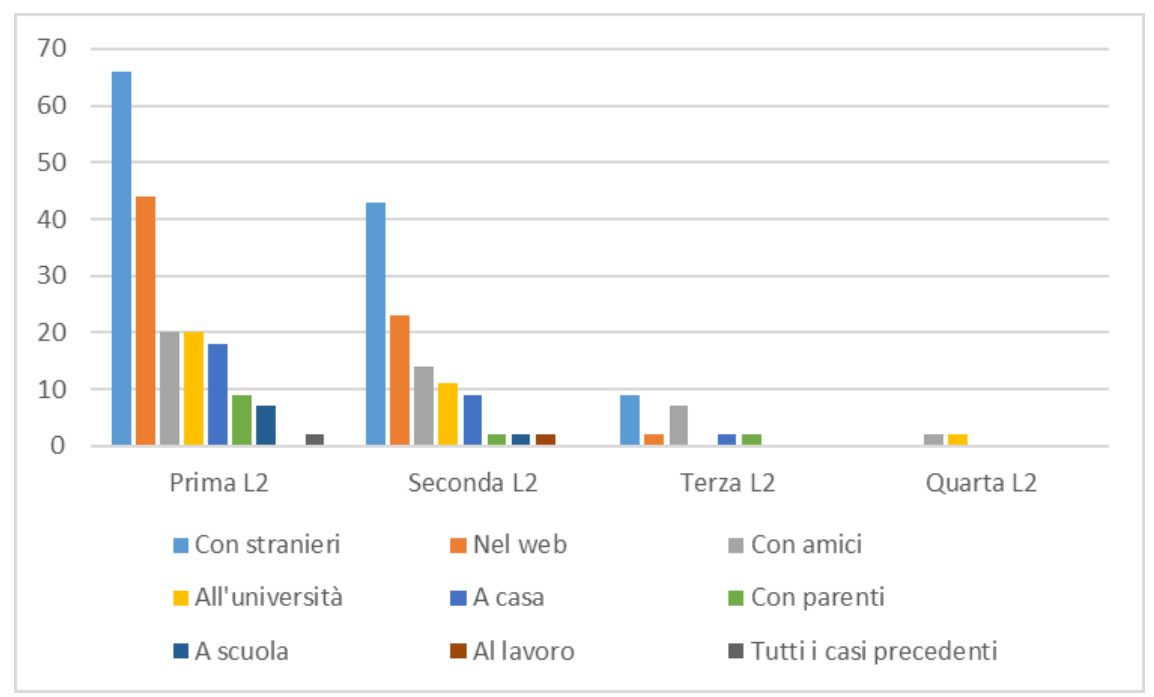

Grafico 4- Distribuzione percentuale dei principali luoghi d'uso e interlocutori tipici

La quinta domanda indagava le modalità di apprendimento delle lingue conosciute. I dati relativi a questa domanda sono informativi: la prima L2 è stata appresa in contesto scolastico dall' $82 \%$ delle rispondenti, il $32 \%$ ha imparato la prima L2 da piccola o in viaggio, il $12 \%$ in famiglia e il $7 \%$ da sola. Anche per la seconda L2 domina l'apprendimento formale (52\% occorrenze), seguito dal contesto informale del viaggio (18\%), dall'apprendimento da piccola (14\% occorrenze), da sola e in famiglia (4\%) e da grande (1 occorrenza). La terza e la quarta L2, invece, sono solo il frutto dell'apprendimento in contesto informale.

L'ultima domanda voleva far riflettere sulla competenza comunicativa e rilevare la percezione delle rispondenti sulla propria padronanza linguistica. In termini assoluti la prima L2 è conosciuta a livelli di competenza intermedia 
(41\%) e medio/bassa (sommando elementare e principiante con il 39\%); il livello avanzato è dichiarato solo dal $14 \%$ delle studentesse. Anche per la seconda L2 prevalgono le dichiarazioni circa una competenza intermedia (29\%) o generalmente medio-bassa (25\%). Per la terza L2 la competenza è decisamente più bassa; il 16\% delle rispondenti sceglie principiante-elementare, in un caso livello intermedio e in uno da madrelingua.

È interessante l'ordine di presentazione delle lingue conosciute che, contrariamente alle aspettative, non è stato quello relativo al livello di competenza/ padronanza nella lingua. Cerchiamo di fare alcune ipotesi e confrontiamole con i dati raccolti: l'ordine gerarchico delle L2 potrebbe rispondere a criteri di ordine temporale (la prima L2 appresa), di rilevanza d'uso, di prestigio. Per lo più, dai dati registrati e dalle risposte sugli usi linguistici dichiarati, sembra che il prestigio e la dimensione affettiva alla lingua (maggiore uso, vicinanza emotiva) siano le principali motivazioni che giustificano l'ordine delle lingue scelto. Sembrano dunque prevalere criteri quali la frequenza d'uso e, appunto, le dimensioni affettive.

$\grave{\mathrm{E}}$ importante anche sottolineare che le lingue apprese in contesto formale e non formale registrano una bassa frequenza d'uso e una percezione di competenza che si colloca tra livello principiante e elementare. Come prevedibile, oltre alla lingua madre, le lingue apprese in contesto famigliare sono principalmente i dialetti. Tra le lingue acquisite in modo informale, lo spagnolo risulta essere la lingua maggiormente appresa, in particolare in autonomia (in viaggio o da autodidatta) in cui le apprendenti riescono a raggiungere discreti risultati di competenza.

Con la formulazione di questi quesiti, ci interessava da un lato studiare il primo contatto dell'apprendente con la lingua distinguendo un apprendimento esperienziale, da uno formale, non formale e informale (Rogers, 2014; Schugurensky, 2000) e dall'altro la percezione di adeguatezza o meno rispetto alle competenze linguistiche di ogni lingua segnalata. Durante i loro studi accademici, le rispondenti saranno chiamate in diverse occasioni a autovalutare le proprie produzioni, è così importante osservare come rappresentino le proprie competenze linguistiche.

\section{Le pratiche di scrittura}

Nella sezione $C$ del questionario si chiedeva di descrivere le proprie pratiche di scrittura da più prospettive, declinandole in base a scopi, destinatari e situazioni di comunicazione, cioè di rappresentarle in termini quantitativi, situazionali, qualitativi, contenutistici, funzionali e dei riceventi tipici.

Nel quantificare le proprie attività di scrittura complessive, il 36\% del campione dichiara di scrivere almeno 500 parole al giorno, il 21\% afferma di scrivere almeno 1500 parole al giorno e poco meno di un sesto (16\%) indica almeno 2500 parole scritte al giorno. Di contro, un quarto delle intervistate non arriva a scrivere 500 parole in una settimana e una matricola riconosce di non scrivere regolarmente. 
Nella seconda domanda, era richiesto di esplicitare le occasioni in cui ricorrono le attività di scrittura. Ne erano proposte quattro, due situazioni interattive di cui una più informale e una formale (cioè comunicazioni con segreterie e docenti) e due occasioni in cui la scrittura assolve anche compiti cognitivi profondi o intimi (studio e introspezione). Prevedibilmente, è preponderante il ricorso alla scrittura per le attività di studio dentro e fuori l'aula, dato che accomuna il 95\% delle rispondenti così come lo è scrivere per comunicare con amici e familiari $(82 \%)$ mentre più della metà comunica con i docenti e il personale universitario in forma scritta $(66 \%)$. Poco più dei due quinti, infine, ricorre alle potenzialità trasformative dello scrivere praticando attività autoriflessive private su emozioni e pensieri.

Definire le proprie pratiche di scrittura in termini di tipi testuali significa riflettere sulle forme di comunicazione scritta praticate in riferimento ai limiti o alle opportunità presupposti dalle diverse condizioni di mezzo, profondità e ampiezza, intenzioni dell'emittente e aspettative del ricevente, margini di libertà espressiva e stilistica ecc. Tra le forme proposte, alcune rientrano nell'ambito della scrittura breve o "spedita" (Pistolesi, 2004) e sono tendenzialmente meno controllate e di registro colloquiale. Tra queste, predominano i messaggi digitali brevi (sms, post ecc.), praticati dal $98 \%$ delle rispondenti, cui seguono le lettere ed e-mail informali (52\%), le cartoline e i bigliettini (43\%), le risposte a questionari (34\%). Nella scrittura formale di tipo funzionale, invece, prevalgono le lettere ed e-mail formali $(73 \%)$ mentre solo la metà dichiara di avere familiarità con la scrittura di tesine e relazioni, cui seguono i temi scolastici (48\%) e testi tipicamente accademici come saggi, articoli e recensioni (32\%). Relativamente poco praticate sono le forme espressive e narrative, con un quarto delle rispondenti che scrive testi di tipo diaristico $(25 \%)$ e il $14 \%$ che scrive testi narrativi.

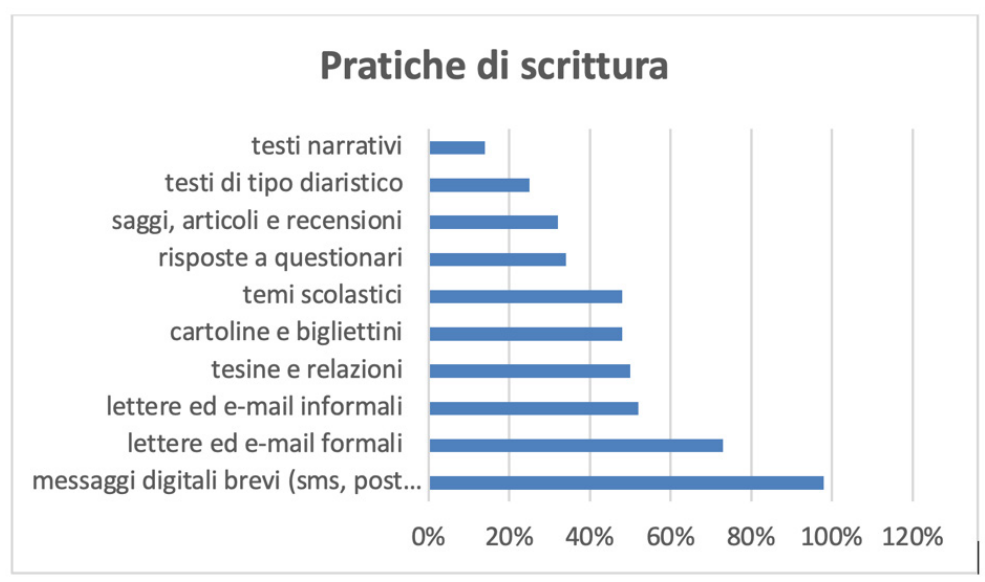

Grafico 5- Testi praticati dalle apprendenti. 
Per vagliare l'ampiezza del repertorio delle singole matricole, è utile considerare la quantità di tipi testuali praticati dalle apprendenti per suddividerli in sottogruppi. Possiamo così notare che il 39\% rientra nel sottogruppo in cui la scrittura è circoscritta a 2-4 tipi di testo; la maggioranza delle rispondenti (52\%) appartiene a quello in cui sono praticate da 5 a 7 tipi testuali e solo il 9\% dichiara pratiche di scrittura molto variate a livello testuale (8-10 tipi).

La domanda successiva, che verteva sui contenuti di scrittura, evidenzia l'atteso predominio delle risposte relative agli argomenti di studio e/o lavoro (98\%). Anche qui, è pertinente focalizzare l'attenzione sul ventaglio di argomenti trattati, riflessione che permette di notare come, per il 36\% delle rispondenti, gli argomenti di studio siano gli unici di cui si scrive, a fronte di un $7 \%$ che dichiara di scrivere unicamente di sentimenti ed emozioni. Suddividendo le scelte proposte in base alle categorie "scrittura funzionale" e "scrittura privata", infine, si può notare che poco meno della metà delle rispondenti $(48 \%)$ dichiara di scrivere in riferimento a entrambi i sottogruppi.

Considerando gli scopi sociali, espressivi o funzionali per cui possono essere praticate, risultano familiari le forme di scrittura con un forte valore di rielaborazione cognitiva e mnemonica ( $86 \%$ delle matricole), così come sono prevalenti gli altri scopi ricorrenti nella scrittura funzionale (informare, 75\%; raccontare, $61 \%$; descrivere, $59 \%$ ). Le forme meno praticate sono quelle di scrittura persuasiva $(25 \%)$, di relazione amicale e socializzazione $(25 \%)$, con intenti ludici e di intrattenimento (20\%) o di tipo intimista e introspettivo (18\%).

Con la domanda successiva, si rilevava la varietà dei destinatari tipici e si invitava a riflettere sulle differenze comunicative proponendo situazioni di comunicazione simmetrica o asimmetrica, familiare e poco controllata o pianificata e sostenuta, ristretta a pochi destinatari noti o rivolta a un pubblico ampio di riceventi non identificati. Le risposte indicano l'ampiezza delle occasioni di comunicazione simmetrica tra pari $(86 \%)$ o comunque con persone della cerchia familiare (64\%), affettiva e sociale (32\%). Tra le possibili occasioni di comunicazione asimmetrica, pianificata e sostenuta, prevedibilmente, è la comunicazione con docenti e personale universitario a rendere conto delle esperienze del $45 \%$ delle rispondenti e in soli due casi la comunicazione scritta avviene anche con pubblici ampi e indifferenziati.

\section{Scrittura e riflessività}

L'ultima sezione del questionario era incentrata sull'analisi riflessiva nelle attività di scrittura ricorrenti. La quasi totalità delle domande era a risposta aperta così da lasciare ampia possibilità di formulazione e poter rilevare le categorie utilizzate dalle rispondenti.

La prima domanda, di tipo generale, chiedeva di indicare il livello di soddisfazione rispetto ai testi prodotti. La maggioranza delle rispondenti si dichiara "abbastanza soddisfatta" (57\%) rispetto a un $23 \%$ che è "molto soddisfatto" e a un $18 \%$ che è solitamente "poco soddisfatto" da quanto scrive. Solo in un caso, il grado di soddisfazione è massimo. 
Le risposte alla richiesta di indicare che cosa risulti più o meno convincente nei testi scritti possono essere raggruppate in 5 categorie: ideazione dei contenuti; testualità; elementi microlinguistici; esecuzione; aspetti psicologici o affettivi. Tra i punti di forza dichiarati, prevalgono gli aspetti relativi alla testualità (come scorrevolezza, coerenza e organizzazione discorsiva, capacità di sintesi ecc., con un $27 \%$ di risposte) seguiti da aspetti riconducibili a stati d'animo o facoltà dello spirito (come sincerità, impegno, fantasia, capacità ironica o di esprimere emozioni, senso di benessere nello scrivere ecc., con un $18 \%$ di risposte) e, con pari percentuale ( $14 \%$ di risposte), da elementi microlinguistici (come correttezza grammaticale, repertorio lessicale di base, punteggiatura, ortografia e sintassi) e aspetti dell'ideazione e pianificazione del testo (quali chiarezza, ricchezza e originalità dei contenuti). In ultimo, sono segnalati tratti della capacità manuale e della presentazione visiva (come calligrafia e scrittura ordinata, con il 9\%).

Rispetto ai punti deboli, le segnalazioni preponderanti, con il $41 \%$, riguardano gli aspetti microlinguistici (come povertà lessicale, difficoltà verbali, sintattiche, ortografiche e di punteggiatura) e, a seguire con il 36\%, gli elementi testuali (quali scrittura prolissa o involuta, testi macchinosi, poco scorrevoli, poco coerenti o comprensibili). Con la stessa percentuale complessiva (7\%) seguono sia le segnalazioni relative all'esecuzione (es., lentezza dovuta a scarsa pratica scrittoria) sia quelle riguardanti la dimensione psicologica (problemi di fantasia, ripetitività e paura di sbagliare).

Alla richiesta di precisare le difficoltà incontrate, il 48\% delle rispondenti indica problemi di sintassi, incertezze grammaticali ecc., con una netta prevalenza delle segnalazioni su difficoltà lessicali di base e terminologiche (23\%) mentre il 36\% delle risposte riporta problemi macrolinguistici con segnalazioni sulla complessità del lavoro di sintesi $(16 \%)$ e, in subordine, di tenuta di un livello espressivo adeguato (11\%). Gli impedimenti a livello affettivo (11\%) riguardano principalmente l'effetto demotivante e di disimpegno che può risultare dal dover scrivere di contenuti considerati poco interessanti mentre le indicazioni sulle difficoltà di esecuzione riguardano aspetti quali la gestione del tempo negli esami $(7 \%)$ e l'ideazione $(4 \%)$.

\begin{tabular}{|l|l|l|}
\hline Testi da scrivere & facili & difficili \\
\hline risposte a questionari (Q) & $91 \%$ & $9 \%$ \\
\hline pagine di diario (D) & $89 \%$ & $11 \%$ \\
\hline lettere ed e-mail formali (LE) & $70 \%$ & $30 \%$ \\
\hline narrazioni (N) & $55 \%$ & $45 \%$ \\
\hline presentazioni, recensioni di libri, progetti ecc. (PR) & $41 \%$ & $59 \%$ \\
\hline saggi brevi e tesine (ST) & $39 \%$ & $61 \%$ \\
\hline resoconti e relazioni (RR) & $30 \%$ & $70 \%$ \\
\hline
\end{tabular}

Tabella 2-Compiti di scrittura e difficoltà percepita (in ordine crescente). 
Con le domande 4 (a risposta chiusa) e 5, si entrava nella discussione di dettaglio giacché si chiedeva di definire sette forme testuali come facili o difficili da scrivere e poi di motivare la risposta. L'elenco proposto comprendeva una selezione di testi distintivi dell'ambito accademico e di uso comune quali lettere ed e-mail formali (LE); resoconti e relazioni (RR); saggi brevi e tesine $(\mathrm{ST})$; presentazioni e recensioni di libri, progetti ecc. (PR); risposte a questionari $(\mathrm{Q})$; narrazioni $(\mathrm{N})$; pagine di diario $(\mathrm{D})$.

Per la difficoltà percepita, primeggiano i resoconti e le relazioni $(70 \%)$, i saggi brevi e le tesine (61\%), le presentazioni e le recensioni di libri, progetti ecc. (59\%). A seguire, le narrazioni sono considerate impegnative dal 45\% delle rispondenti e le lettere ed e-mail formali dal 30\%. La scrittura diaristica e le risposte a questionari sono generalmente considerate, per quanto non unanimemente, di facile scrittura (11\% e 9\%). Per cogliere il carico cognitivo dato dall'insieme dei testi che le matricole faticano a gestire nello studio universitario, è utile analizzare i dati disaggregati. Ne risulta che, per il 50\% delle matricole, 3 o 4 delle forme testuali proposte sono complesse da scrivere, poco meno della metà $(43 \%)$ considera difficili soltanto 1 o 2 tipi di testo e una ridotta percentuale $(7 \%)$ dichiara difficoltà nello scrivere la gran parte $(5$ o 6) delle forme testuali ricorrenti nello studio accademico.

Alla richiesta di spiegare l'origine delle difficoltà espresse, le rispondenti le riconducono a carenze individuali (39\%, es. q. 34 "Trovo difficoltà nella scrittura formale, non padroneggio un lessico adatto per lettere ed e-mail formali; nei saggi bravi e nelle tesine la mia difficoltà è quella di sviluppare un testo correttamente suddiviso per argomenti e tesi da sviluppare."), alle difficoltà intrinseche alla scrittura di determinati tipi testuali (32\%), all'assenza di formazione o di pratica adeguata (27\%).

Dovendo indicare come le difficoltà incontrate sono generalmente affrontate o superate, molte risposte (32\%) appaiono generiche e relative alla sfera psicologica e affettiva, informative solo sul coinvolgimento e la motivazione delle rispondenti. Il 68\% delle matricole, invece, fornisce indicazioni sulle strategie di superamento degli ostacoli e le capacità metacognitive di potenziamento delle attività di scrittura a fini di studio. Le pratiche ricorrenti sono descrivibili secondo tre categorie: l'uso di strumenti cartacei e digitali per il controllo o l'ampliamento lessicale (20\%); il ricorso a un giudizio/aiuto esperto esterno $(36 \%$, in cui si predilige la consultazione tra pari, con affini e familiari e in un solo caso si interpellano i docenti) e l'attivazione di procedure che mirano a regolare e migliorare il processo e i prodotti della scrittura (55\%). Queste ultime, quando sono specificate, includono il lavoro di documentazione su esempi e modelli a cui ispirarsi $(14 \%)$ e la schematizzazione dei testi da comprendere o da scrivere (7\%). È interessante notare come, a livello individuale, solo il $18 \%$ delle matricole segnali un repertorio di strategie diversificato, con il ricorso ad almeno due delle strategie proposte.

Focalizzando l'attenzione sul processo più che sul prodotto della scrittura, la domanda 7 chiedeva di precisare com'è organizzato il lavoro di produzione dei testi scritti per studio. Scrive di getto il 32\% delle rispondenti, di contro, 
il 50\% dichiara di passare attraverso più fasi di ideazione e ri/definizione dei contenuti e il $16 \%$ delle matricole, che si comporta diversamente a seconda dei casi, dimostra un'apprezzabile sensibilità alla dimensione pragmatica della scrittura e alla necessaria attenzione a fattori quali scopi, destinatari, contenuti, tipi di testo ecc. su cui definire il piano d'azione scrittoria. In un caso, infine, una studentessa non madrelingua dichiara di tradurre in italiano quanto scriverebbe in L1, con le ricadute che possiamo immaginare sui tempi di esecuzione, in termini di accuratezza microlinguistica e di adeguatezza macrolinguistica.

Le ultime domande interrogavano i bisogni formativi percepiti in rapporto ad abilità e contenuti considerati necessari o utili nel corso OFA. Nell' $11 \%$ dei casi, si esprime una generica speranza di poter migliorare la propria capacità di scrittura mentre nel $9 \%$ si auspica un lavoro sui metodi di studio. Più nello specifico, il 18\% delle rispondenti propone obiettivi di lavoro su aspetti microlinguistici (in particolare sulla grammatica e il lessico di base), e il 36\% segnala esigenze formative su aspetti macrolinguistici (specie la scrittura di sintesi, i testi argomentativi, la comprensione scritta e la tenuta di registri formali). Nel $20 \%$ dei casi, le indicazioni riguardano entrambi i livelli di intervento. Tra i casi restanti, infine, è interessante notare le risposte focalizzate sugli esami in sé, che evidenziano, sì, una motivazione strumentale, ma denotano anche una riflessione metalinguistica sul test di accesso iniziale o sui "criteri valutativi di sistema" (q. 18, "Dato che frequentiamo il corso OFA perché non siamo riusciti a pieno a raggiungere il punteggio minimo, penso che sia giusto affrontare argomenti come sinonimi, punteggiatura e un po' di grammatica in generale. (visto che gli argomenti nel test erano questi)") o all'esame finale per assolvere il debito formativo (q. 42, "capire come si svolgerà la prova per esercitarmi e riuscire a superarla, non ho degli argomenti specifici", q. 42).

\section{Discussione}

Nell'ambito delle indagini sulle competenze linguistiche degli studenti universitari in Italia, ci siamo interrogate sul rapporto con la scrittura e su come e quanto le pratiche di comunicazione scritta caratterizzino la popolazione giovanile per individuarne dei tratti distintivi e rapportarli agli usi scritti ricorrenti nella comunità di pratica accademica. Le percentuali fornite non hanno valore statistico giacché non si tratta di questionari anonimi, il campione discusso è ristretto alle studentesse frequentanti un corso OFA di due corsi di laurea $\mathrm{e}$ la rilevazione è limitata all'anno accademico 2018/2019. Con questi limiti e senza pretese di rappresentatività, possiamo sviluppare alcune riflessioni utili sui punti di interesse: il profilo delle apprendenti in esame e le loro autodefinizioni in termini di biografia linguistica, di pratiche scrittorie, di "bisogni linguistico-comunicativi" espressi sulla scrittura, e di senso di autoefficacia percepita circa i prodotti richiesti in ambito accademico.

Riguardo alla dimensione plurilingue delle rispondenti, i dati rappresentano una gamma di lingue e culture variegata e complessa, ma registrano anche il progressivo abbandono delle lingue apprese in contesto formale. La lingua 
francese, spagnola e tedesca, acquisite in genere nella scuola secondaria di I e II grado, non sono usate in contesto accademico e sono relegate a obiettivi comunicativi privati, legati all'oralità (comunicazione con persone straniere, spesso in viaggio) o alla velocità di fruizione (ascoltare musica, comunicare nel web, vedere serie tv). L'uso dell'inglese condivide le stesse caratteristiche, ma se ne attestano anche impieghi più accademici (studio e test di idoneità). Alcune L2 (in particolare lo spagnolo), infine, sono apprese anche in età adulta, in contesti informali (in viaggio) o in autoapprendimento, con risultati di competenza discreti.

Circa gli apprendimenti linguistici a scuola, si osserva come una focalizzazione circoscritta alla competenza linguistica e poco incentrata sulle occasioni d'uso comunicativo mostri fragilità allorché le lingue non evolvono e si atrofizzano nel tempo. Questi dati sottolineano l'importanza di una formazione linguistica mirata non solo allo sviluppo di competenze linguistiche tout court ma tesa anche alla crescita di competenze comunicative e pragmatiche in una relazione dinamica con le realtà interne ed esterne al contesto scolastico. In un'università che, come principio teorico, vuole internazionalizzarsi offrendo corsi in altre lingue e richiedendo una competenza plurilingue come requisito di accesso, ${ }^{6}$ però, il plurilinguismo individuale non appare valorizzato: il profilo linguistico delle apprendenti plurilingui per cui l'italiano non è la L1 e alle quali è stato attribuito l'OFA emerge soltanto in termini di deficit nelle competenze linguistiche presupposte in italiano L1.

Sugli usi linguistici situati nelle attività di produzione/interazione scritta $\mathrm{e}$ il grado di familiarità con generi e tipi di testo praticati in ambito accademico, alcuni risultati dell'indagine appaiono significativi. I dati sulle pratiche di scrittura indicano che sono solitamente poco estese e circoscritte al solo ambito di studio. Circa la dimensione sociale e situazionale delle comunicazioni in ambito accademico, il passaggio a un maggior grado di formalità atteso e il più ampio ruolo comunicativo assolto dalla comunicazione scritta nelle università sono prevedibilmente parte del divario tra le richieste della scuola superiore e dell'università. Di certo, i dati indicano uno stacco esperienziale che riguarda non soltanto i contenuti (che rappresentano lo "specifico formativo" della specializzazione insita negli studi universitari), ma anche e soprattutto il piano funzionale (scopi della scrittura) e del pubblico a cui ci si rivolge. Sul piano qualitativo, invece, se globalmente la gamma dei tipi di testo praticati appare sufficientemente estesa per più della metà delle rispondenti, si rileva che, per il $55 \%$ delle studentesse, le forme considerate difficili comprendono solo esempi di scrittura funzionale nell'ambito accademico e che il 59\% dichiara una formazione o una pratica ridotta o nulla.

Riguardo alle origini delle difficoltà e alla loro percezione, rileviamo la riflessività dimostrata su prospettive diverse rispetto alle incompetenze personali dichiarate. Vista la dimensione formativa dell'indagine e le ricadute previste sulla definizione del corso in termini di motivazione, sillabo e

6. Nei corsi di laurea qui considerati, è richiesto un livello A2 di inglese. 
coinvolgimento nel lavoro previsto, i punti trattati nel questionario si ponevano come una serie di domande tese a sollecitare la riflessione sul rapporto con le lingue note e con le abitudini di scrittura per definire il proprio profilo di apprendente rispetto alle abilità attese nel contesto accademico. Dalle richieste di autovalutazione sulle competenze linguistiche in sé alle domande più specifiche sulle forme testuali ricorrenti nei percorsi di studio intrapresi, i quesiti proposti e le risposte fornite tracciavano un "percorso riflessivo" che si è poi rispecchiato nella prospettiva da cui le matricole hanno risposto alle ultime domande aperte. Qui, la riflessione si è focalizzata su alcune delle coordinate che caratterizzano la scrittura come sistema integrato di azioni e fasi che precedono o seguono la stesura dei testi. È interessante notare come emerga sempre più rilevante la dimensione della "grammatica dei testi" e di quella microlinguistica vista in un'ottica non più assoluta, ma situata. Definire le proprie pratiche di scrittura in termini di tipi di testo significa riflettere sulle forme di comunicazione scritta praticate in riferimento ai limiti o alle opportunità presupposti dalle diverse condizioni di mezzo, profondità e ampiezza, intenzioni dell'emittente e aspettative del ricevente, margini di libertà espressiva e stilistica ecc. Nel contesto specifico, ciò ha significato considerare le proprie attività di scrittura in riferimento a modelli testuali codificati in una determinata comunità linguistica e (ri)costruire il panorama linguistico personale vagliandone le declinazioni e i (nuovi) obiettivi funzionali prevalenti. $\mathrm{Ne}$ risulta un quadro complessivo in cui è predominante il ruolo educativo che la formazione universitaria svolge o dovrebbe svolgere anche e soprattutto rispetto a un rapporto più consapevole con le forme e le funzioni che la scrittura incarna in contesto accademico (e professionale).

\section{Conclusioni}

Considerando in ottica longitudinale i fenomeni di decadimento linguistico osservabili nel passaggio ai diversi ordini scolastici, De Mauro (De Mauro, 2001 (2018) invitava a riflettere e a ridefinire la formazione linguistica rapportandola alla progressione dei cicli di istruzione. Si tratta, dunque, di affiancare all'attenzione posta sulla lingua "oggetto di acquisizione" (e sul livello di padronanza atteso al termine della scuola superiore) l'attenzione alla capacità comunicativa, vista in rapporto all'apprendente che ne esperisce le condizioni d'uso nel nuovo contesto della comunicazione accademica (e disciplinare).

Circa la scrittura e la relativa formazione, negli anni, l'attenzione degli studiosi si è spostata dal talento individuale al prodotto di scrittura, dal processo di produzione al complesso sistema cognitivo e di strutturazione delle conoscenze messo in atto dalla scrittura stessa. Nella pratica didattica, ciò ha portato a critiche argomentate circa la tradizionale tendenza a richiedere di produrre testi scritti senza la necessaria pratica sulle diverse fasi insite nella stesura di un buon testo scritto o nell'analisi linguistica, sociolinguistica e pragmatica di una varietà di tipi e modelli testuali. Questo processo attivo implica sia capacità manuali sia capacità metacognitive, cognitive e linguistiche, necessarie per 
saper ideare, organizzare e produrre un messaggio in relazione ai parametri di definizione di una comunicazione efficace. Ciò presuppone anche una diversa prospettiva sugli apprendenti in cui la loro voce assuma un valore superiore all'essere fonte di dati evidenziali e li veda attori nel processo di acquisizione degli strumenti necessari ad agire nelle diverse forme dell'espressione e della comunicazione accademica (cfr. Fielding, 2001, Bloemert et al., 2020).

Lo spostamento del focus sull'apprendente implica che sia valorizzata la dimensione riflessiva in cui poter verbalizzare il proprio rapporto con la scrittura per poterlo interrogare e ridefinire nella consapevolezza degli usi accademici attesi e per potervi sviluppare una propria voce autonoma.

Riguardo alle competenze linguistiche viste nel loro insieme, il "profilo medio" che emerge dai dati della nostra indagine è quello di un'apprendente il cui repertorio di conoscenze plurilingui è sviluppato in famiglia oppure deve molto, nel bene e nel male, alla formazione scolastica e alle occasioni di un'acquisizione spontanea e informale.

Rispetto al rapporto con la scrittura, naturalmente, parliamo di un campione di studentesse che non ha conseguito il punteggio minimo nella prova di selezione, ma parliamo anche di apprendenti che risultano poco familiari con le specificità della lettura e della scrittura all'università. In proposito, ci siamo chieste se non sia preferibile, per l'intera popolazione studentesca, prevedere una dimensione formativa in cui gli apprendenti abbiano la possibilità di interrogarsi e appropriarsi delle forme di comunicazione scritte e orali ricorrenti nei corsi di studio universitario intrapresi. In una simile prospettiva, un rapporto consapevole con la scrittura da parte degli studenti rappresenta un prerequisito importante di una didattica della scrittura mirata allo sviluppo di quelle competenze accademiche trasversali che dovrebbero favorire una costruzione critica del sapere universitario. L'ottica della formazione linguistica universitaria di tipo rimediale appare invece negare nei fatti la natura situata di tali apprendimenti e ne presuppone una visione statica in cui l'acquisizione è in sé data e predeterminata.

Circa le pratiche di scrittura, i dati mostrano l'intrinseca eterogeneità che caratterizza questo pur piccolo campione di apprendenti allorché si rilevano le differenze rispetto alle abitudini di scrittura e al repertorio di tipi testuali con cui hanno familiarità. Nonostante questo, ciò che accomuna le nostre apprendenti è il forte legame che associa le attività di scrittura allo studio universitario, ambito a cui rimandano le occasioni, gli scopi e i destinatari delle loro produzioni scritte di tipo funzionale e formale.

I nostri dati suffragano dunque la necessità di operare nel senso di una ridefinizione della formazione linguistica in contesto accademico: questa dovrebbe essere concepita in una prospettiva di tipo evolutivo e non di tipo episodico, rimediale o compensativo. In altre parole, incentivare un accrescimento continuo delle conoscenze linguistiche a livello universitario implica di promuovere un apprendimento mirato a "funzionalizzare i vari ambiti disciplinari nell'acquisizione di usi linguistici nuovi e diversi" (Voghera et al., 2009: 107), cioè potenziare tutte le capacità dei giovani di muoversi e agire consapevolmente 
nello "spazio linguistico universitario" e nelle sue diverse coniugazioni disciplinari. È un'ottica che impone, da un canto, di interrogarsi sulla realtà educativa universitaria, sulle capacità di comprensione/produzione che questa richiede alle giovani generazioni e sui loro bisogni formativi e, dall'altro, di indagare sulla conoscenza e rappresentazione di tale realtà da parte dei giovani apprendenti nonché sulla loro percezione circa le risorse di cui dispongono e di cui necessitano nell'affrontare il percorso universitario. Tutto ciò al fine di un'assunzione di responsabilità rispetto agli obiettivi di crescita necessaria per poter sviluppare l'ampiezza e la profondità linguistico-comunicativa richieste dal sapere accademico. Sono obiettivi di lifelong learning che dovrebbero concretizzarsi in percorsi di apprendimento in cui le eventuali "fragilità" individuali possano essere riconosciute, comprese e risolte nell'ottica strutturale di una comunità di pratica accademica.

\section{Bibliografia}

Andorno, C. (2014), Una semplice informalità? Le e-mail di studenti a docenti universitari come apprendistato di registri formali. In Cerruti, M. \& Corino, E. \& Onesti, C. (Edd.), Lingue in contesto. Studi di linguistica e glottodidattica sulla variazione diafasica. (pp. 13-32). Alessandria: Edizioni dell'Orso.

Barré-De Miniac C. (2002). Le rapport à l'écriture. Une notion à plusieurs dimensions. In Pratiques : linguistique, littérature, didactique, 113-114, 29-40.

Barré-De Miniac C. (2003). La littéracie:au-delà du mot, une notion qui ouvre un champ de recherches variées. In Schweizerische Zeitschrift für Bildungswissenschaften 25 (1), 111-123.

Bloemert, J. \& Paran, A. \& Jansen, E. (2020) Connecting students and researchers: the secondary school student's voice in foreign language education research. Cambridge Journal of Education, 50:4, 429-449.

Brusco, S. \& Lucisano, P. \& Salerni, A. \& Sposetti, P. (2014). Le scritture degli studenti laureati: un'analisi di prove di accesso alla Laurea Magistrale. In Colombo, A. \& Pallotti, G. (Edd.). Litaliano per capire (pp. 147-165). Roma: Aracne.

Cacchione, A. \& Rossi, L. (2016). La lingua troppo (росо) variabile: monolinguismo e mistilinguismo in testi funzionali di matricole universitarie. In Ruffino, G. \& Castiglione, M. (Edd.). La lingua variabile nei testi letterari, artistici e funzionali contemporanei: analisi, interpretazione, traduzione. Atti del XIII Congresso SILFI (pp. 457-489). Firenze: Franco Cesati.

Caffi, C. (1991). Aspetti pragmatici e testuali delle introduzioni a tesi di laurea e specializzazione in materie scientifiche. In Lavinio, C. \& Sobrero, A. (Edd.). La lingua degli studenti universitari (pp. 71-98). Firenze: La Nuova Italia.

Calamai, S. (2012). Per un'analisi delle competenze scrittorie negli studenti universitari. In Bernini, G. \& Lavinio, C. \& Valentini. A. \& Voghera, M. (Edd.). Atti dell'1 $1{ }^{\circ}$ Congresso dell'associazione italiana di linguistica applicata. Competenze e formazione linguistiche. In memoria di Monica Berretta (pp. 77-99). Perugia: Guerra.

Ciliberti, A. \& Anderson, L. (Edd). (2006). Le forme della comunicazione accademica. Ricerche linguistiche sulla didattica universitaria in ambito umanistico. Milano: FrancoAngeli. 
Cognigni, E. (2014). L'autobiografia linguistica a scuola tra plurilinguismo e affettività: dal ritratto al racconto delle lingue. In CROSSROADS: Languages in (E) motion (p.189-200). Napoli: Photo City Edizioni-University Press.

Coonan, C. M. \& Bier, A. \& Ballarin, E. (Edd). (2018). La didattica delle lingue nel nuovo millennio. Le sfide dell'internazionalizzazione. Atti del IV Congresso della società di Didattica delle Lingue e Linguistica Educativa DILLE (Università Ca' Foscari Venezia, 2-4 febbraio 2017). Venezia: Edizioni Cà Foscari.

Council of Europe (2019b). European language portfolio. Disponibile su htttps://www. coe.int/en/web/portfolio.

De Santis, C. \& Fiorentino, G. (2018). La carica dei 600: la campagna mediatica sul declino della lingua italiana. Circula. Rivista di ideologie linguistiche, 7, 2-28.

Desideri, P. \& Tessuto, G. (2011). Il discorso accademico. Lingue e pratiche disciplinari. Urbino: Quattroventi.

Desoutter, C. (2021), Il rapporto con la scrittura: un elemento costitutivo della competenza di scrittura in L1 e in L2. in In Grassi, R. (Ed.) La scrittura per l'apprendimento dell'italiano L2 (pp. 19-35). Firenze: Franco Cesati editore.

Fielding, M. (2001). Students as radical agents of change. Journal of Educational Change, 2, 123-141.

Fiorentino, G. (Ed.). (2009). Perché la grammatica? La didattica dellitaliano tra scuola e università. Roma: Carocci.

Fiorentino, G. (2015). Aspetti problematici del discorso accademico: un'analisi dei riassunti delle tesi di laurea. Cuadernos de Filología Italiana, 22, 263-284.

Fusari, S. \& Luporini, A. (2016). La comunicazione tra studenti e docenti via forum e e-mail: strategie di cortesia. In Bianchi, F. \& Leone, P. (Edd.) Studi AItLA 4: Linguaggio e apprendi- mento linguistico. Metodi e strumenti tecnologici. (pp. 51-66). Milano: Officinaventuno.

Grandi, N. (2018). Sulla penetrazione di tratti neo-standard nell'italiano degli studenti universitari. Primi risultati di un'indagine empirica. Griseldaonline, 17(1). https:// doi.org/10.6092/issn.1721-4777/9021

Grassi, R. \& Nuzzo. E. (2012). Le (in)competenze di scrittura all'università: evidenze dai test di valutazione iniziale. In Bernini, G. \& Lavinio, C. \& Valentini, A. \& Voghera, M. (Edd.), Atti dell'1 $1^{\circ}$ Congresso dell'associazione italiana di linguistica applicata. Competenze e formazione linguistiche. In memoria di Monica Berretta (pp. 101-118). Perugia: Guerra.

Grassi, R. (Ed.) (2021). La scrittura per l'apprendimento dell'italiano L2. Firenze: Franco Cesati editore.

Gohard-Radenkovic A., \& Rachédi, L. (2009). Récits de vie, récits de langues et mobilités. Nouveaux territoires intimes, nouveaux passages vers l'altérité. Paris: L'Harmattan.

Guerriero, A. R. (Ed.) (2021). Scrivere. Idee per la didattica della scrittura. Firenze: Franco Cesati editore.

Guerriero, A. R. (2021). Introduzione. In Guerriero, A. R. (Ed.) (2021). Scrivere. Idee per la didattica della scrittura. (pp. 21-22). Firenze: Franco Cesati editore.

Kramsch, C. (2004). Language, thought, and culture. In A. Davies, \& C. Elder (Edd.), The handbook of applied linguistics (pp. 235-265). London/New York: Blackwell Publishers. doi:10.1002/9780470757000.ch9

Lambert, P. (2005). Les répertoires plurilectaux de jeunes filles d'un lycée professionnel: une approche sociolinguistique. (Tesi di dottorato, Université Stendhal, Grenoble). Disponibile su 154 Carnets d'Atelier de Sociolinguistique ${ }^{\circ} 4$ ethnographique, thèse de doctorat, Université Stendhal, Grenoble. 
Lavinio, C. \& Sobrero, A. (Edd.). (1991). La lingua degli studenti universitari. Firenze: La Nuova Italia.

Lavinio, C. (1991). I problemi della scrittura. In Lavinio, C. \& Sobrero, A. (Edd.). La lingua degli studenti universitari. (pp. 13-30). Firenze: La Nuova Italia.

Lavinio, C. (1998). Lingue speciali e tipi di testo, tra argomentazione, esposizione e descrizione. In Pavesi, M. \& Bernini, G. (Edd), Le lingue speciali all'Università. Atti del convegno di Pavia. 28-29 ottobre 1996 (pp. 143-171). Roma: Bulzoni.

Lavinio, C. (2011). (In) competenze metalinguistiche di base in laureati in Lettere. Italiano Lingua Due, 3, 2. 258-291. Disponibile su https://riviste.unimi.it/index. $\mathrm{php} / \mathrm{promoitals/article/view/1925}$

Levy, D. (2009). Soi et les langues. In Levy D. \& Kramsch C. ,\& Zarate G. (Edd.). (2009). Précis du plurilinguisme et du pluriculturalisme (pp. 69-81). Paris: Éditions des archives.contemporaines.

Lo Duca, M. G. (2017). Lettera in risposta alla 'proposta' dei 600. Disponibile su http:// www.societadilinguisticaitaliana.net

Lubello, S. (2019). L'italiano scritto accademico all'università tra L1 e L2: riflessioni e proposte per un curricolo. Testi e linguaggi, 19, 178-189.

Lubello, S. \& Nobili, C. (2019). Coretto... ma non troppo: analisi della competenza valutativa di testi scritti in un esperimento trasversale in italiano L1/LS. In Palermo, M. \& Salvatore, E. (Edd.). Scrivere nella scuola di oggi. Obiettivi, metodi, esperienze (pp. 267-278). Firenze: Franco Cesati.

Lüdi, G. (2005). L'intérêt épistémologique de l'autobiographie linguistique pour l'acquisition / enseignement des langues. In Mochet ,\& al. (Edd.). Plurilinguisme et apprentissages, Mélanges Daniel Coste (pp. 143-154). Lyon: ENS Editions.

Martari, Y. (2019). Italiano L2 di italiani? Varietà di apprendimento di scriventi in italiano lingua madre e lingua seconda. In Carbonara, V. \& Cosenza, L. \& Masillo. P. \& Salvati, L. \& Scibetta, A. (Edd.). Il parlato e lo scritto: aspetti teorici e didattici. (pp. 137-154). Pisa: Pacini.

Molinié, M. (Ed.). (2006). Biographie langagière et apprentissage plurilingue. Le français dans le monde, Recherches et applications (39). Paris: CLE International.

Pagliara, F. (2018). L'analisi dei bisogni nel TBLT: un caso di studio sull'italiano come lingua della comunicazione accademica. In Cortés Velásquez, D. \& Nuzzo, E. (Edd.). Il task nell'insegnamento delle lingue. Percorsi tra ricerca e didattica al CLA di Roma Tre. (pp. 77-104). Roma: Roma Tre Press.

Parlamento europeo, Consiglio dell'Unione europea (2006). Raccomandazione del Parlamento europeo e del Consiglio del 18 dicembre 2006 relativa a competenze chiave per l'apprendimento permanente (2006/962/ CE) Disponibile su https:// goo.gl/zOEmdC

Perregaux, C. (2007). Avec le biographique, développer l'intérêt pour la complexité des répertoirs langagiers dans la formation et la recherche. In Verdelhan-Bourgade M. (Ed). Le français langue seconde: un concept et des pratiques en évolution (pp. 143-156). Bruxelles: De Boeck.

Piemontese, M. E. (2002 (2021), La scrittura: un caso di problem solving. In Guerriero, A. R. (Ed.) (2021). Scrivere. Idee per la didattica della scrittura. (pp. 23-35). Firenze: Franco Cesati editore.

Pineau, G. (1983). Produire sa vie: autoformation et autobiographie. Montréal/Paris: Édilig/Albert St-Martin.

Pugliese, R. \& Della Putta, P. (2017). Il mio ragazzo è italiano B1. Sulle competenze di scrittura formale degli studenti universitari. Lend, XLVI, 4, 83-110. 
Pugliese, R. \& Della Putta, P. (2020), Il discorso accademico scritto degli studenti universitari nelle prove di esame: un confronto tra italiano L1 e L2. Italiano LinguaDue, n. 1, 26-41.

Pugliese, R. (2021), Scrivere all'università: ricerche, applicazioni, prospettive. In Grassi, R. (Ed.) La scrittura per l'apprendimento dell'italiano L2 (pp. 171-207). Firenze: Franco Cesati editore.

Rogers, A. (2014). The Base of the Iceberg: Informal Learning and Its Impact on Formal and Non-formal Learning. Berlin/Toronto: Opladen/Verlag Barbara Budrich.

Santipolo, M. (2002). Dalla sociolinguistica alla glottodidattica. Torino: Utet Libreria.

Schugurensky, D. (2000). The forms of informal learning: towards a conceptualization of the field, in NALL Working Paper, 19. Ontario Institute for Studies in Education of the University of Toronto: Centre for the Study of Education and Work, Department of Sociology and Equity Studies in Education.

Sobrero, A. (1991). Prefazione. In Lavinio, C. \& Sobrero, A. (Edd.). La lingua degli studenti universitari. (pp. 1-9). Firenze: La Nuova Italia.

Sofia V. \& Favero, E. (2018). L' autobiografia linguistica nella pratica didattica. Una proposta per valorizzare la madrelingua e il plurilinguismo. Latina: Aracne.

Sposetti. P. (2008). Litaliano degli studenti universitari. Roma: Homolegens.

Valentini, A. (2002). Tratti standard (e neostandard) nell'italiano scritto di studenti universitari. Linguistica e Filologia, 7, 303-322.

Van den Avenne, C. (Ed). (2005). Mobilité et contacts de langues. Paris: L'Harmattan.

Viale, M. (2011). Le competenze grammaticali di base per l'università: dati da un'esperienza didattica. In Corrà, L. \& Paschetto, W. (Edd.). Grammatica a scuola. (pp. 137-149). Milano: FrancoAngeli.

Voghera, M. \& Giordano, R. \& Guerriero, A. R. (2009). Grammatica e matricole: proposte di educazione linguistica. In Fiorentino, G. (Ed.). Perché la grammatica? La didattica dell'italiano tra scuola e università. (pp. 93-108). Roma: Carocci. 